\title{
Ueber die Beziehungen der quergestreiften Muskeln zum Papillärkörper der Lippenhaut.
}

\author{
Von
}

Dr. med. W. Podwyssozki (jun.),

Privat-Docenten d. Allg. Pathologie an d. militär.-merlicin. Akademie zu St. Petersburg.

Hierzu Tafel XVII.

Vorliegende Mittheilung bat den Zweck, einige Lücken in unseren Kenntnissen über die histologischen Verhältnisse der quergestreiften Muskeln der Haut im Allgemeinen und speciell der Lippenhaut anszufüllen. Gleichzeitig dürften die mitzutheilenden Tbatsachen von Interesse und nicht unwichtig für die noch immer streitige Frage über das Verhalten der Muskelfasern zu den Sehnenfasern sein.

Alles, was gegenwärtig über die Hautmuskeln in der histologischen Literatur vorliegt, bezieht sich eigentlich auf die glatte Musknlatur; die quergestreiften bleiben, meines Wissens, soviel wie gar nicht berücksichtigt. „Willkürliche quergestreifte Muskelfasern gelangen nur im Gesicht, am Bart und an der Nase von der Tiefe in die Haut hinein und endigen in der Lederbaut bald unter schiefem Winkel, bald senkrecht zwischen den Haaren und Talgdrüsen gelegen." Diese von Biesiadecki ${ }^{1}$ ) noch im Jahre 1871 gemachte Beschreibung entspricht auch heutzutage, wenn wir von A eby's mehr topographischen Angaben abseben, unseren Kenntnissen über die Verhältnisse der quergestreiften Muskeln zu der Haut. Die feineren histologischen Beziehungen sowie die Art der Befestigung der Muskeln bleiben unbekannt.

In den entsprechenden Capiteln der gegenwärtig ziemlich umfangreichen Literatur liber die Anatomie und Histologie der Haut wird auch beute noch einfach erwähnt, dass an man-

1) Stricker's Handbuch d. Gewebelehre. Bd. I, p. 599. 
chen Stellen der Haut des Gesichts sich quergestreifte Muskelfasern aus der Tiefe bis zum Corium erstrecken, an welchem sie sich dureh Bindegewebe inseriren. Was die Mundmuskulatur speciell betrifft, so drücken sich einzelne Autoren, auf Grund ihrer mikroskopischen Untersuchung in der Weise ans, das einige Muskeln wie zygomaticus, risorius, rectus labii sup. et inf. ${ }^{1}$ ) ( $A \in b y$ ) und quadratus sup. et inferior theils in der Haut, theils in der Schleimhaut an verschiedenen Stellen der Lippe endigen (A eby²), W. Krause $)^{3}$ ). Allein die Art und Weise dieser Endigung, sowie das Verhalten einzelner Muskeltasern im Papillarkörper ist zur Zeit nicht näher bekannt.

Dank der fixirenden Wirkungen der sogenannten starken Flemming'schen Flüssigkeit (Chromosmiumsäure-Gemisch), gelang es mir, einige feinere Verhältnisse der quergestreiften Muskeln zu der papillaren Schicht der Haut an gewissen Stellen der Mundspalte des Kaninchens festzustellen, nämlich an den an die äusseren resp. behaarten Theile angrenzenden Bezirken der Schleimhaut des Lippenrandes, vorzugsweise aber an den beiden polsterartigen, parallel dem Lippenrande sich erstreckenden Erhabenheiten der Schleimbaut, welche zu beiden Seiten der Oberlippe liegen. An den behaarten Theilen des Lippenrandes sind die betreffenden Verhältnisse nicht mehr so scharf ausgeprägt. Weniger deutlich ist die mitzutheilende Art der Endigung der Muskeln am Lippenrande des Meerschweinchens ausgeprägt. Bei anderen Thieren, wie Ratte, Maus, Katze, Hund, sowie beim Menschen, konnte ich an der Mundmuskulatur einen so engen Zusammenhang der Muskeln mit dem Epithel wie beim Kaninchen nicht verfolgen.

An einer Reihe von in concentrirter wässriger Safranin-Lösung und nachfolgend in schwacher alcoholischer Picrinsäure-Lösung. gefärbten und nach dem bekannten Hermann-Flemming'schen Verfabren behandelten sagittalen Schnitten kleiner Stückehen des Lippenrandes, welche vorher gut in starker Chromosmiumessig-

1) Oder musc. labii proprius nach W. $\mathrm{Krause}$, oder compressor labii nach Klein.

2) Chr. Aeby, Die Muskulatur der menschlichen Mundspalte (Arch. f. mikr. Anatomie Bd. XVI, 1879, p. 659-662).

3) W. Krause, Allg. und mikrosk. Anatomie. Nachtrag. 1881, p. 68. 
säure-Gemische fixirt, ausgewaschen und in Alcohol gehärtet worden waren, fällt bei mikroskopischer Untersuchung folgendes, deutlich ausgeprägtes Bild in die Augen:

Von der Tiefe des Unterhautgewebes erheben sich zur Schleimhaut-Oberfläche mehr oder minder parallel mit einander verlaufende einzelne Btindel quergestreifter Muskelfasern. Entweder nabe an der Grenze der epithelialen Schleimbaut-Schicht, oder etwas von derselben entfernt, beginnt ein Zerfall der Bündel in feinere bis zu einzelnen Muskelfasern, welche sich entweder in Bïndelchen von mehreren primitiven Muskel-Fibrillen oder aber in einzelne primitive Fibrillen zerlegen. Infolge eines solchen allmählich fortschreitenden Zerfalls bildet $\operatorname{sich}$ aus jedem ursprünglichen dickeren Muskel-Bündel ein mehr oder minder breit ausgedehntes pinselartiges Gebilde; die Elemente dieses Pinsels bestehen aus einzelnen Muskelfibrillen (vergl. Fig. 1 und Fig. 5). Die Querstreifung der letzteren ist vollkommen deutlich schon mit einer Vergrösserung von $1 / 250$ zu unterscheiden.

Die in der beschriebenen Weise entstandenen feineren Fibrillenbiindelchen und einzelnen Muskelfibrillen nehmen, an der Grenze der epithelialen Schleimschicht angelangt, verschiedene Richtungen ein, aber immer richten sie sich zu ihrem Endziel, zum Epithel. Durch Kreuzung der durch die erwähnte Zerspaltung entstandenen zahlreichen Glieder eines jeden dickeren Muskelfasern-Biindels mit ähnlichen Gliedern aus den benachbarten Muskel-Bündeln, entsteht an der Grenze des Papillarkörpers ein ganzes System von zablreichen Muskelfibrillen-Bündelchen und von einzelnen Muskel-Fibrillen (Fig. 1). Während einzelne dieser Fibrillen mehr in senkrechter Richtung direct zum Epithel verlaufen, nehmen andere eine grössere Ablenkung von der ursprünglichen Richtung des Stammbtindels, um erst nach einem mehr schrägen Verlauf durch die Grenztheile des Unterzellhautgewebes zum Epithel zu gelangen. Hier gehen die Fibrillen-Bündel sowie die einzelnen Fibrillen in homogene, glänzende, feine sehnenartige Fasern uber, vermittelst welcher sie sich im Stratum mucosum befestigen. Die erwähnten Netze verdanken ihre Enstehung mehr der Durchkreuzung der ebenerwäbnten sehnenartigen Fortsätze, als der Kreuzung der Muskelfibrillen selbst.

An manchen Stellen findet man eine sehr eigenthümliche Beziehung der Muskelfibrillen-Bündelchen und der pri- 
mitiven Muskelfibrillen zu den in's Unterzellhautgewebe hineinragenden epithelialen interpapillären Wülsten (Fig. 1, 2, 5, 6). Fast jeder dieser Wülste besitzt ein ihm entsprechendes Muskel-Bündel, dessen aus dem Zerfall gebildete Glieder vorzugsweise ihm angehören resp. den Wulst umfassen und in dessen Substanz endigen (vergl. Fig. 2, 5, 6). Ich sage vorzugsweise, da einzelne Fibrillen auch zu den nachbarlichen Wülsten gelangen, was die Ursache der schon geschilderten Netzbildung unter dem Stratum mucosum ist.

Wenn man ferner das Verhalten der Muskelfasern und Muskelfibrillen zu dem Epithel untersucht, so findet man einen noch engeren Zusammenhang des Muskelsystems mit dem epithelialen. An manchen Stellen nämlich unterscheidet man sehr klar ein Ein'dringen von einzelnen Muskelfibrill-Bündelchen, mit deutlicher Querstreifung, in die Papillen hinein und sogar in die entfernteste Spitze derselben. Dieses Verbalten ist schon ganz deutlich mit einer schwachen Vergrösserung (60-80) wahrzunehmen (vgl. Fig. 1). Mit starken Systemen verfolgt man natürlich deutlichere Bilder (vergl. Fig. 3, 4, 6).

Die Anordnung der Muskelfibrillen-Bündel und deren weiterer Zerfall im Bereiche der Papille bleibt iuberall dieselbe: sie halten sich immer an der Oberfläche der Papillen resp. baften an den Grenzflächen der interpapillaren epithelialen Wülste.

Sehr schöne und belehrende Bilder des Verbaltens der Muskeln in den Papillen bekommt man an denjenigen Papillen, welche in ihrem grösseren Durchmesser durchgeschnitten sind und eine Capillarschlinge enthalten (Fig. 6). Am nächsten kornmt das Muskelgewebe der Gefässschlinge an der Basis der Papille, wo die beiden (auf dem Schnitte) gegenüberliegenden interpapillären Wülste sich einander nähern und den engeren Theil oder den Hals der Papille bilden. An einigen Papillen ist der Hals so schmal, dass die emporsteigenden Muskelfibrillen der Gefässschlinge (Capillare) eng anliegen und sie umringen. Es ist kein seltener Befund, dass am Papillenhalse die Muskelbtindelchen einander überkreuzen (vgl. Fig. 3 und 4).

Wie endigen nun die Muskeln am Epithel? Diese Frage kann nur zum Theil und zwar durch die Untersuchung der entsprechenden Stellen mit den stärksten Linsen beantwortet werden. Wenn man mit einem $1 / 18-1 / 20$ Oelimmersions-Systeme von 
Z eiss verschiedene Stellen der Präparate durchmustert, so unterscheidet man Folgendes: Die feinsten sehnenartigen Fibrillen, welche Fortsätze der primitiven Muskelfibrillen sind, kommen in innigste Verbindung mit dem Epithel des Stratrum mucosum. Das ist sicher! - Es handelt sich nicht bloss um eine Berührung einzelner Fibrillen mit dem epithelialen Gewebe, sondern vielleicht um ein Eindringen derselben in dessen Substanz, nämlich in die intercellulären Spalten. Was dies Verhalten zu den intercellulären Spalten anlangt, so kann ich mich leider nicht bestimmter darüber äussern, da hier selbst die stärksten Vergrösserungen im Stich liessen. Es ist aber sicher, dass wir es hier nicht mit einer einfachen Berïhrung, einem einfachen Nebeneinanderliegen zi thun haben, sondern mit einer viel complicirteren Verbindung. An manchen Stellen macht es den Eindruck, als ob die Sehnenfäserchen mit der Basilar-Membran des Stratum mucosum zusammenflössen (vergleiche Fig. 2-6). Diese letztere scheint keine structurlose Membran zu sein, sondern stellt vielleicht ein engmaschiges fibrilläres Netz dar ${ }^{1}$ ). Die sehnenartigen Fäserchen der Muskelfibrillen dürften mit den Fibrillen dieses netzartigen Saumes sich vereinigen.

Die Präparate, nach welchen die oben stehende Beschreibung gemacht ist, geben gute Gelegenheit, eine wichtige und noch immer offene Frage aus der Histologie des Muskelsystems zu beantworten; ich meine den Uebergang der Muskelsubstanz in die Sehnens ubstanz.

Die Anschauungen der Autoren spalten sich betreffs dieses Gegenstandes, wie bekannt, in zwei Hauptrichtungen. Nach

1) Die fibrilläre Structur der Membrana propria der Drüsen-Alveolen wurde von mir im Jahre 1882 für die Bauchspeicheldrüse genau nachgewiesen (Beiträgez. Kenntniss d. feineren Baues der Bauchspeicheldrüse. Arch. f. mikroskopische Anatomie Bd. XXI, p. 768. Die entsprechende Zeichnung ist in der entsprechenden russischen Arbeit gegeben). Auf Grund vieler histologischer Angaben bin ich geneigt anzunehmen, dass überall, wo eine Membrana basilaris, welche das epitheliale Gewebe von dem Bindegewebe abgrenzt, vorhanden ist (Haut, Darm, Drüsen-Alveolen u. s. w.), dieselbe keine structurlose Membran, sondern ein engpfadiger, fibrillärer, netzartiger Saum ist. 


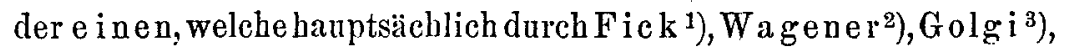
theilweise auch durch $\mathrm{K} \ddot{o l l i k e r}{ }^{4}$ ) vertreten ist, besteht zwischen Muskel- und Sehnenfibrillen eine Continuität, ein unmittelbarer Uebergang. Nach Wagener und besonders nach Golgi bilden nicht blos die dickeren Sehnenfasern eine direkte Fortsetzung der Muskelfasern, sondern es gilt dies auch für die primitiven Sehnenund Muskelfibrillen ,una non interrotta continuazione delle fibrille di cui appare constituita la fibra muscolare primitiva nelle fibrille dalla cui unione sono constituiti i dendinetti primitivi" (Golgi pag. 9). Golgi führt, mit vollem Rechte, seinen Befund des unmittelbaren anatomischen Ueberganges (per continuitatem) der Muskelfasern in die Sebnenfasern als neuen Beweis der engen embryonalen Verwand tschaftzwischen den Muskeln und dem Bindegewebe an.

Nach der zweiten Richtung, an welche die Mehrzahl der A utoren sich hält (Häckel ${ }^{5}$ ), Herzig6), Biesiadecki7), Weismann $\left.^{8}\right)$, Rollet $^{9}$ ), Ranivier ${ }^{10}$ ), W.Krause ${ }^{11}$ ), H.Frey ${ }^{12}$ ), Toldt ${ }^{13}$ ) und andere) besteht zwischen den Muskel- und Sehnenfasern nur eine Contiguität, aber kein unmittelbarer Zusammenhang, und ob-

1) A. Fick, Ueber die Anheftung d. Muskelfasern an die Schnen (Arch. f. Anatomie, Physiologie und wiss. Medicin, 1856, p. 425).

2) G. R. Wagener, Ueber die Muskelfasern der Evertebraten (Reichert's Archiv 1863, p. 221). - Ueber einige Erscheinungen an den Muskeln lebendiger Corethra plumicornis-Larven (Arch. f. mikr. Anat. Bd. X, 1874, p. 297).

3) C. Golgi, Contribuzione alla istologia dei muscoli volontari. Milano 1880. - Annotazioni interno all' istologia normale e patologica deimuscolivolontari. Con 2 Tavolo. (Sep.-Abdr. aus d. Arch. p. le scienze mediche Vol. II. Nr. 11, 1881, p. 8-11.)

4) A. Kölli k e r, Handb. d. Gewebelehre. Leipzig 1852, p. 172.

5) Hä c k e l, Canstatt's Jahresbericht 1857.

6) H erzig, Wiener Sitzungsberichte Bd. XXX, 1858, p. 73.

7) A. B i esiad e cki und Her $z$ ig, Ibidem Bd. XXXIII, 1858, p. 148.

8) We is m a n n, Zeitschrift f. rat. Medicin Bd. XII, 1861, p. 126.

9) Rollet, Wiener Sitzungsber. Bd. XXI, 1856.

10) L. Ra n vie r, Traité technique d'Histologie. 1878, p. 503 . - Leçons d'Anatomie génér. s. 1. systeme Musculaire 1880, p. 245.

11) W. Kra us e, Allgem. und mikroskop. Anatomie 1876, p. 82.

12) H. Frey, Handb. d. Histologie 1876, 5. Auflage.

13) C. Toldt, Gewebelehre 1877, p. 183. 
wohl dié eine an der anderen fest angeheftet ist, so sind beide nur durch eine Kittsubstanz, welche man lösen kann, verlöthet.

Meine Präparate sprechen ganz entschieden für die erste Meinung; sie bieten einen evidenten Beweis des unmittelbaren Ueberganges der Muskelfasern in die Sehnenfasern und, was noch wichtiger ist, der directen Continuität $z w$ ischen den primitiven Muskel- und Sehnenfibrillen. Die früher beschriebene pinselartige Zerspaltung eines Muskelfasers in Muskelfibrillen-Bündelchen und ferner in einzelne Muskelfibrillen, welche in Sehnenfibrillen unmittelbar übergehen, giebt die beste Gelegenheit in situ zu sehen, ohne Anwendung irgend welcher dissociirenden Mittel, dass wirklich die Sehnenfasern in ihren feinsten Gliedern resp. Fibrillen eine directe Fortsetzung der Muskelfasern und Muskelfibrillen darstellen (vergleiche Fig. 2-6).

An manchen primitiven Muskelfibrillen unterscheidet man das allmähliche Verschwinden der Querstreifung und den Uebergang der quergestreiften Fibrille in ein homogenes, glänzendes, nicht gestreiftes Sebnen-Fäserchen. Dieser Uebergang erinnert sehr an die von $W$ age $n \in r^{1}$ ) beschriebenen und gezeichneten Verhältnisse. Mit den stärksten Linsen ist es unmöglich irgend welche Vereinigungslinie hier zu finden, die auf eine Kittsubstanz zu beziehen wäre.

Eins möchte ich Betreffs dieser Frage noch bemerken. Das genannte Volumen nämlich der aus einer Muskelfaser stammenden Sehnenfäserchen ist immer kleiner als das Volumen der Muskelfibrillen der entsprechenden Muskelfaser. Dieser Umstand, welcher schon aus dem einfachen Vergleiche der gesammten Sehnenfäserchen einer Muskelfaser mit der Muskelfaser selbst evident ist, bekommt eine vollkommene Bestätigung bei der genauen Betrachtung der Uebergangsstelle eines Muskelfibrillen-Bündelchens in die entsprechenden Sehnenfibrillen (vergl. Fig. 5). Sehr oft sieht man eine einzelne Muskelfibrille in eine einzige Sehnenfibrille übergehen; es ist aber nicht selten, dass ein ganzes Muskelfibrillen-Bündelchen, aus $4-5$ primitiven Fibrillen bestehend, nur in $2-3$ Sehnenfibrillen iibergeht. In den Muskelfibrillen-Bündelchen sind es die peripheren Schichten desselben, welche, wie es scheint, in die Sehnenfasern

1) G. R. Wagener, Archiv f. mikroskop. Anatomie Bd. X, 1874, p. 297. 
übergehen. Das Verhalten des Sarcolemma's lässt sich nicht deutlich erkennen.

An der Stelle des pinselartigen Zerfalls einer Muskelfaser in Muskelfibrillen-Bündelchen, sowie in einzelne primitive Fibrillen findet immer eine mehr oder minder grosse Anbäufung von Muskelkernen Statt, ein Befund, welcher, nach den ähnlichen Ermittelungen von Frorie ${ }^{1}$ ) bei den Muskeln der Amphibien und Sängethiere, als allgemeine Erscheinung an der Uebergangsstelle der Muskelfasern in die Sehnenfasern angenommen werden kann. An vielen Stellen findet man bisweilen Muskelkerne an dem Uebergange feiner Maskelfibrillen-Bündelchen in Sehnenfasern und zwar anch im Bereiche der Papillen, wenn dort Muskel-Bündelchen hineindringen (vergl. Fig. 3-4).

Wenn wir jetzt nach der pbysiologischen Bedeutung der beschriebenen Beziehungen der quergestreiften, willkürlichen Muskeln zum Papillenkörper fragen wollten, so ist sie zuerst wahrscheinlich mit der Mimik der Lippen verbunden, welche bekanntlich beim K̇aninchen so stark ausgebildet und so vollkommen ist. Ganz so wie mit Zügen kann das Thier mit der beschriebenen Muskeln den epithelialen Belag der Lippen und zwar die kleinsten Bezirke derselben beherrschen, ein Umstand welcher fiir die mimischen Funktionen augenscheinlich von der grössten Wichtigkeit ist. Ausser einer solchen psycho-physiologischen Rolle der erwähnten Muskelbefestigung dürfen wir noch eine andere, rein physiologische annehmen. Durch die Befestigung einzelner Muskelfasern an den interpapillären epithelialen Wülsten, sowie durch das Eindringen der einzelnen Muskelfibrillen-Bündelchen in die Papillen binein kann während der Thätigkeit der Muskeln eine indirecte vasomotorische Wirkung auf die Capillaren der Papillen ausgeiibt werden, indem durch die relative Verschiebung der interpapillären epithelialen Wülste eine kleinere oder grössere Blutfüllung der in den Papill verlaufenden Capillaren hervorgerufen wird. Was für einen $Z$ weck eine solche indirecte vasomotorische Wirkung der quergestreiften Muskeln haben könnte, bleibt freilich zur Zeit unbekannt.

1) A. Froriep, Ueberdas Sarcolemm uud die Muskelkerne (Arch. f. Anatomie und Physiologie. Anat. Abth. 1878, p. 425-427). 


\section{Erklärung der Abbildnngen anf Tafel XVII.}

Alle Figuren sind nach Präparaten gezeichnet, welche aus den erwähnten polsterartigen Erhabenheiten der Schleimhaut beim Kaninchen genommen sind. Färbung bei Fig. 1 und Fig. 5 mit Safranin; bei Fig. 2, 3, 4, 6 mit Safranin und nachfolgend mit schwacher alkoholischer Pikrinsäurelösung.

Fig. 1. Beziehungen der quergestreiften Muskelfasern, Muskelfibrillenbündelchen und Muskelfibrillen zum Papillarkörper. Netzartige Kreuzung der Glieder der nachbarlichen Muskelfasern an der Grenze des Stratum mucosum and des Bindegewebes. Eindringen einzelner Muskelfibrillenbündelchen in die Papillen. (Hartnak Obj. 5, Ocul, 2.)

Fig. 2. Umfassung eines interpapillären epithelialen Wulstes mit Muskelfibrillenbündelchen; Uebergang einzelner Muskelfibrillen in die Membrana basilaris des Epithels. (Z eiss Oel-Imm. System 1/18, Ocul. 2.)

Fig. 3 und 4. Eindringen vieler Muskelfibrillenbündelchen in eine Papille. Absonderung einzelner Muskelfibrillen von dem Bündelchen im Bereiche der Papillen und Uebergang dieser Fibrillen zum Epithel. Scheinbares Zusammenfliessen einzelner Sehnenfibrillen mit der Membrana basilaris des Stratum mucosum. (Fig. 3 gezeichnet mit Zeis s Oel-Imm. System 1/1s, Ocul. 2; Fig. 4 mit Oel-Imm. System $Z$ eiss $1 / 18$, Ocul. 3.)

Fig. 5. Pinselartiger Zerfall einer Muskelfaser in einzelne Muskelfibrillenbündelchen und fernerer Zerfall dieser letzteren in einzelne Muskelfibrillen. Unmittelbarer Uebergang der Muskelfibrillen in Sehnenfibrillen. (Zeiss Oel-Imm. System 1/18, Ocul. 2.)

Fig. 6. Umfassung eines interpapillären Wulstes mit den Zerfallsgliedern einer Muskelfaser. Eindringen eines Muskelfibrillenbündelchens in eine Papille unmittelbar neben einem Capillargefässe. (Zeis s OelImm. System 1/18, Ocul. 2.) 